Revista de Matemática: Teoría y Aplicaciones 4(2): 1-11 (1997)

\title{
SOBRE LA CONTINUIDAD DE LA PROYECCIÓN MÉTRICA ${ }^{1}$
}

\author{
José R. Morales ${ }^{2}$
}

\begin{abstract}
Resumen
En esta nota mostraremos que la proyección métrica asociada a subespacios de Chebyshev en espacios de Banach que poseen la propiedad $(\omega M)$ es una aplicación continua.
\end{abstract}

\section{Introducción}

Iniciaremos el desarrollo del presente artículo dando a conocer la notación que usaremos, recordar ciertas definiciones y propiedades que usaremos en el transcurso del mismo.

Sea $(E,\|\cdot\|)$ un espacio de Banach. Por $S_{E}\left(B_{E}\right)$ denotamos la esfera unitaria (bola unitaria) de $E$, respectivamente.

En el año 1936, J. A. Clarkson (ver [6]) introdujo los espacios de Banach Uniformemente Convexos en la forma siguiente:

Definición 1.1 Un espacio de Banach E se dice que es Uniformemente Convexo, [(UR), Uniformemente Rotundo], si para cada $0<\epsilon \leq 2$ existe un $\delta=\delta(\epsilon)>0$ tal que para todo,

$$
x, y \in S_{E} \quad y \quad\|x-y\| \geq \epsilon \text { entonces } \quad\left\|\frac{x+y}{2}\right\|<1-\delta(\epsilon) .
$$

Ahora, pasamos a recordar la noción, los espacios de Banach estrictamente convexos.

Definición 1.2 Un espacio de Banach $E$ se dice que es estrictamente convexo, [(R), rotundo], si para todo, $x, y \in S_{E} y\|x+y\|=2$ entonces $x=y$.

En el año 1955, fuerón introducidas dos generalizaciones de los espacios (UR); una de carácter local, los espacios Localmente Uniformemente Convexos, introducidos por A. R. Lovaglia (ver [6]) y la otra generalización es de carácter Uniforme, los espacios (KR), definidos por K. Fan - I. Glicksberg (ver [6]).

\footnotetext{
${ }^{1}$ Este trabajo fue financiado por el Proyecto CDCHT - C - 551 - 92

${ }^{2}$ Universidad de los Andes, Facultad de Ciencias, Departamento de Matemáticas, Grupo de Análisis Funcional, MÉrida, Venezuela
} 
Definición 1.3 Un espacio de Banach $E$ se dice que es Localmente Uniformemente Convexo, [(LUR) Localmente Uniformemente Rotundo], si dados $x \in S_{E} y \epsilon>0$, existe un $\delta=\delta(\epsilon, x)>0$, tales que para todo $y \in S_{E}$, con

$$
\|x-y\| \geq \epsilon \quad \text { entonces } \quad\left\|\frac{x+y}{2}\right\|<1-\delta .
$$

Es claro que,

$$
U R \Rightarrow L U R \Rightarrow R
$$

Definición 1.4 Sea $k \geq 2$ un entero. Decimos que el espacio de Banach $E$ es un espacio $K R$ si para cualquier sucesión $\left(x_{n}\right) \subset B_{E}$ tal que,

$$
\lim _{n_{1}, \ldots, n_{k} \rightarrow \infty}\left\|x_{n_{1}}+\ldots+x_{n_{k}}\right\|=k
$$

entonces, $\left(x_{n}\right)$ es una sucesión de Cauchy en E.

En 1991, Bor - Luh - lin y Wenyao Zhang (ver [5]) introdujerón los espacios $\omega$ R que claramente generalizan los espacios KR.

Definición 1.5 Sea $E$ un espacio de Banach. Se dice que $E$ es un espacio $\omega R$, si para cualquier sucesión $\left(x_{n}\right) \subset B_{E}$ tal que

$$
\lim _{n_{1}, \ldots, n_{k} \rightarrow \infty}\left\|x_{n_{1}}+\ldots+x_{n_{k}}\right\|=k_{1} \quad \forall k \in \mathbb{N},
$$

entonces, $\left(x_{n}\right)$ es convergente en $E$.

En 1988, Nan - Chao Xun y Wang - Jian Hua (ver [13]) introdujerón los espacios L-KR, $K \geq 1$ que generalizan los espacios LUR y localizan los espacios KR.

Definición 1.6 Sea $k \geq 1$ un estero. Un espacio de Banach $E$ se dice que es un espacio $L-K R$, si para cada sucesión $\left(x_{n}\right) \subset B_{E} y x \in S_{E}$, tales que

$$
\lim _{n_{1}, \ldots, n_{k} \rightarrow \infty}\left\|x_{n_{1}}+\ldots+x_{n_{k}}\right\|=k+1
$$

entonces

$$
\lim _{n \rightarrow \infty}\left\|x_{n}-x\right\|=0
$$

En [13] los autores extienden la anterior definición e introducen los espacios L $\omega \mathrm{R}$, que claramente localizan los espacios $\omega \mathrm{R}$.

Definición 1.7 Sea E, un espacio de Banach se dice que $E$ es un espacio LwR si para cualquier sucesión $\left(x_{n}\right) \subset B_{E}$ y todo $x \in S_{E}$, tales que,

$$
\lim _{n_{1}, \ldots, n_{k} \rightarrow \infty}\left\|x+\sum_{i=1}^{k} x_{n_{i}}\right\|=k+1 \quad \forall k \in \mathbb{N}
$$

entonces

$$
\lim _{n \rightarrow \infty}\left\|x_{n}-x\right\|=0
$$


La relación entre las definiciones anteriores es la siguiente

$$
\begin{aligned}
& U R \Rightarrow 2 R \Rightarrow \cdots \Rightarrow K R \Rightarrow(K+1) R \Rightarrow \cdots \Rightarrow \quad \omega R \\
& \mathrm{y} \quad \Downarrow \quad \Downarrow \quad \Downarrow \quad \Downarrow \quad \forall \quad \forall \\
& L U R \Rightarrow L 2 R \Rightarrow \cdots \Rightarrow L K R \Rightarrow L(K+1) R \Rightarrow \cdots \Rightarrow L \omega R \Rightarrow R
\end{aligned}
$$

En 1979, F. Sullivan (ver [10]) introduce los espacios K-UR y LK-UR, $K \geq 1$ entero, propiedades geométricas que generalizan la noción de Clarkson.

Para $x_{1}, \ldots, x_{k+1} \in E$, se define la función volumen:

$$
V\left(x_{1}, \ldots, x_{k+1}\right)=\sup \left\{\left|\begin{array}{ccc}
1 & \cdots & 1 \\
f_{1}\left(x_{1}\right) & \cdots & f_{1}\left(x_{k+1}\right) \\
\vdots & \ddots & \vdots \\
f_{k}\left(x_{1}\right) & \cdots & f_{k}\left(x_{k+1}\right)
\end{array}\right|: f_{i} \in B_{E^{*}} \quad 1 \leq i \leq k\right\}
$$

y para $i=1, \ldots, k$, sea $d_{i}=d\left(x_{i+1},\left[x_{1}, \ldots, x_{i}\right]\right)$ donde $\left[x_{1}, \ldots, x_{i}\right]$ es el espacio afín generado por $\left\{x_{1}, \ldots, x_{n}\right\}$. Se define

$$
d\left(x_{1}, \ldots, x_{k+1}\right)=\min \left\{d_{1}, \ldots, d_{k}\right\}
$$

y se sabe que, ( ver [14]),

$$
V\left(x_{1}, \ldots, x_{k+1}\right) \rightarrow 0 \Longleftrightarrow d\left(x_{1}, \ldots, x_{k+1}\right) \rightarrow 0 .
$$

Definición 1.8 Sea $k \geq 1$ un entero. Un espacio de Banach $E$ se dice K-Uniformemente Convexo, $(K-U R)$, si para todo $\epsilon>0$ existe un $\delta=\delta(\epsilon)>0$ tal que para todo $x_{i} \in S_{E}, \quad i=$ $1, \ldots, k+1$ y $\frac{1}{k+1}\left\|x_{1}+\ldots+x_{k+1}\right\| \geq 1-\delta$ entonces $V\left(x_{1}, \ldots, x_{k+1}\right)<\epsilon$.

Definición 1.9 Sea $k \geq 1$ un entero. Un espacio de Banach $E$ se dice Localmente $K$ Uniformemente Convexo, (L-KUR). Si para cualquier $\epsilon>0$ y $x \in S_{E}$ existe un $\delta(\epsilon, x)>0$ tal que para

$$
x_{1}, \ldots, x_{k} \in S_{E} \quad y \quad \frac{1}{k+1}\left\|x+x_{1}+\ldots+x_{k}\right\| \geq 1-\delta
$$

entonces $V\left(x, x_{1}, \ldots, x_{k}\right)<\epsilon$.

En [5] los autores logran generalizar las dos nociones dadas anteriormente e introducen los espacios $\omega \mathrm{UR}$ y los espacios L $\omega \mathrm{UR}$.

Definición 1.10 Sea $E$ un espacio de Banach. Se dice que E es un espacio $\omega U R$ si para toda sucesión triangular $\left\{x_{i}^{(n)}: 1 \leq i \leq n, n \in \mathbb{N}\right\}$ en $B_{E}$ con

$$
\lim _{n \rightarrow \infty}\left(n-\left\|\sum_{i=1}^{n} x_{i}^{(n)}\right\|\right)=0
$$

entonces

$$
\lim _{n \rightarrow \infty} d\left(x_{1}^{(n)}, \ldots, x_{n}^{(n)}\right)=0
$$


Definición 1.11 Sea $E$ un espacio de Banach. Se dice que E es un espacio LwUR si para toda sucesión triangular $\left\{x_{i}^{(n)}: 1 \leq i \leq n, n \in \mathbb{N}\right\}$ en $B_{E}$ con

$$
\lim _{n \rightarrow \infty}\left((n+1)-\left\|\sum_{i=1}^{n} x_{i}^{(n)}\right\|\right)=0
$$

entonces

$$
\lim _{n \rightarrow \infty} d\left(x, x_{1}^{(n)}, \ldots, x_{n}^{(n)}\right)=0
$$

También se tiene que,

$$
\begin{aligned}
& U R \Rightarrow 2-U R \Rightarrow \cdots \Rightarrow K-U R \Rightarrow K+1-U R \quad \Rightarrow \cdots \Rightarrow \omega U R \\
& \Downarrow \quad \Downarrow \quad \Downarrow \quad \Downarrow \quad \Downarrow \\
& L U R \Rightarrow L 2-U R \Rightarrow \cdots \Rightarrow L K-U R \Rightarrow L(K+1)-U R \Rightarrow \cdots \Rightarrow L \omega U R \Rightarrow R
\end{aligned}
$$

En 1980, R. Huff (ver [14]) introdujo los espacios casi uniformemente convexos, en la forma siguiente.

Definición 1.12 Sea E un espacio de Banach. Se dice que E es un espacio Casi Uniformemente Convexo, (NUC), si para todo $\epsilon>0$ existe un $\delta>0$ tal que para cada sucesión $\left(x_{n}\right) \subset B_{E}$ con $\operatorname{sep}\left(x_{n}\right) \geq \epsilon$ entonces $C_{o}\left(\left\{x_{n}\right\}\right) \cap(1-\delta) B_{E} \neq \emptyset$, donde

$$
\operatorname{sep}\left(x_{n}\right)=\inf \left\{\left\|x_{n}-x_{m}\right\|: n \neq m\right\}
$$

y $C_{o}(A)$ donde la cápsula convexa es $A$.

D. Kutzarova y Bor - Luh - lin (ver [14]) localizan los espacios (NUC) e introducen los espacios (LNUC).

Definición 1.13 Un espacio de Banach E es llamado Localmente Casi Uniformemente Convexo, (LNUC), si para todo $\epsilon>0$ y cada $x \in S_{E}$ existe un $\delta=\delta(x, \epsilon)>0$ tal que para toda sucesión $\left(x_{n}\right) \subset B_{E} y \operatorname{sep}\left(x_{n}\right)>\epsilon$, entonces

$$
C_{o}\left(\left\{x_{n}\right\}\right) \cap(1-\delta) B_{E} \neq \emptyset .
$$

Es claro, que NUC $\Rightarrow$ LNUC. Es bien conocido, el siguiente resultado, (ver [14]).

$$
\mathrm{L} \omega \mathrm{UR} \Rightarrow \text { LNUC. }
$$

Sea $F$ un subconjunto no - vacio de $E$. Para cualquier $x \in E$ definimos por

$$
d(x, F)=\inf \{\|x-y\| / y \in F\}
$$

la distancia de $x$ a $F$. Para cada $x \in E$, decimos que el punto $y_{0} \in F$ es un punto de mejor aproximación (punto cercano) a $x$ desde $F$ si,

$$
d(x, F)=\left\|x-y_{o}\right\| .
$$


Un subespacio $F$ cerrado de un espacio de Banach $(E,\|\cdot\|)$ se dice que es un conjunto de Chebyshev si para cada $x \in E$ existe un único punto de mejor aproximación a $x$ desde $F$. Uno de los problemas en la teoría de los puntos cercanos es probar la convexidad de un conjunto de Chebyshev en un espacio de Hilbert y caracterizar aquellos espacios de Banach en los cuales todo conjunto de Chebyshev es un conjunto convexo. Una de las formas de atacar tal problema es usando la proyección métrica sobre un espacio de Chebyshev.

Para cada subconjunto $F$ de $E$ y $x \in E$ el conjunto

$$
P_{F}(x)=\{y \in F \mid d(x, F)=\|x-y\|\},
$$

constituye el conjunto de todos los puntos cercanos o de mejor aproximación a $x$ desde $F$. Es claro que $P_{F}(x)$ es un subconjunto cerrado, acotado, que puede ser vacío y es convexo si $F$ lo es. $P_{F}$, es una función multivaluada de $E$ en $F$,

$$
\begin{aligned}
P_{F}: & E \longrightarrow 2^{F} \\
& x \longmapsto P_{F}(x)
\end{aligned}
$$

$P_{F}$ es llamada la proyección métrica sobre $F$. Cuando $F$ es un conjunto de Chebyshev, entonces $P_{F}(x)$ es una función univaluada de $E$ sobre $F$, algunas veces conocida como la aplicación Chebyshev, el operador de mejor aproximación o la función proximidad.

El siguiente resultado nos muestra algunas de las propiedades que posee $P_{F}$. Para una prueba ver [3] y [4].

Lema 1.1 Sean $(E,\|\cdot\|)$ un espacio de Banach y $F \subseteq E$ un subespacio de Chebyshev. Entonces,

1. $P_{F}$, es una aplicación idempotente y tiene gráfico cerrado.

2. Para todo $x \in E$, se tiene

$$
\left\|P_{F}(x)\right\| \leq\left\|x-P_{F}(x)\right\|+\|x\| \leq 2\|x\| .
$$

3. $P_{F}$, es homogénea; esto es, para cada $x \in E$ y $\lambda \in \mathbb{R}$

$$
P_{F}(\lambda x)=\lambda P_{F}(x)
$$

4. $P_{F}$ es aditiva módulo $F$; esto es,

$$
P_{F}(x+y)=P_{F}(x)+P_{F}(y) \text { si } x \in F \text { ó } y \in F,
$$

(en general, $P_{F}$ no es lineal).

5. Para cada $x \in E$,

$$
\left\|x-P_{F}(x)\right\|=d(x, F)=\|x+F\| .
$$

donde,

$$
\|x+F\|=\inf \{\|x+y\|: y \in F\}
$$


Es importante señalar que existen proyecciones métricas que no son aplicaciones continuas. En este sentido sabemos que el primer ejemplo de una proyección métrica discontinua fué provisto por J. Lindeustrauss, ver [4]. Otro ejemplo fue dado por R. Holmes y B. Kripke [12] quienes construyeron un espacio de Banach estrictamente convexo, y no reflexivo poseyendo un subespacio lineal de codimensión 2 cuya proyección métrica es discontinua. Para otros ejemplos de proyecciones métricas discontinuas ver [1] y [4].

Por otra parte, son ampliamente conocidas las condiciones bajo las cuales la proyección métrica es una aplicación continua. En este sentido es importante recordar el siguiente resultado.

Lema 1.2 Sean $(E,\|\cdot\|)$ un espacio de Banach y $F$ un subespacio de Chebyshev de E. $P_{F}$ es continua cuando se cumple una de las siguientes condiciones:

1. F es localmente compacto o

2. $F$ es $\operatorname{dim}(F)<\infty o$

3. E es Uniformemente Convexo;

4. E es un espacio reflexivo, estrictamente convexo y posee la propiedad $(H)$

5. E es un espacio $L 2-U R$

6. E es un espacio $L k-U R$.

Para la prueba de este resultado ver [9], [10], y [11].

En [3] y [9] encontramos ciertos resultados que nos muestran que la continuidad de la proyección métrica puede ser usada para demostrar la convexidad de conjuntos de Chebyshev.

En la próxima sección generalizamos los resultados del Lema [1.2] (4) y (5), los cuales fueron dados por F. Sullivan [10] y Yu Xintai [11] respectivamente.

\section{Resultado Principal}

Esta sección la iniciaremos recordando, ciertas propiedades geométricas de los espacios de Banach y luego daremos nuestro principal resultado. En 1975, B. B. Panda y O. P. Kapoor (ver [7]) introdujerón la propiedad (M), en la forma siguiente.

Definición 2.1 Un Espacio de Banach E se dice que posee la propiedad (M) si para cada $x \in S_{E}$ y cada sucesión $\left(x_{n}\right) \subset B_{E}$ tal que,

$$
\lim _{n \rightarrow \infty}\left\|x_{n}+x\right\|=2
$$

entonces $\left(x_{n}\right)$ es compacto en $B_{E}$. 
El autor en [7] logró generalizar la anterior propiedad como sigue.

Definición 2.2 Sea $k \geq 1$ un esntero. Un Espacio de Banach $E$ se dice que posee la propiedad (K-M) si para cada $x \in S_{E}$ y cada sucesión $\left(x_{n}\right) \subset B_{E}$ tal que,

$$
\lim _{n_{1} \ldots n_{k} \rightarrow \infty}\left\|x+\sum_{i=1}^{k} x_{n_{i}}\right\|=k+1
$$

entonces $\left(x_{n}\right)$ es compacto en $B_{E}$.

Es claro, que si en la definición 2.2 tomamos $k=1$, entonces se obtiene la definición 2.1 esto es,

$$
\text { Propiedad (1-M) } \Longleftrightarrow \text { Propiedad (M) }
$$

y

$$
\text { Propiedad }(\mathrm{M})+\mathrm{R} \Longleftrightarrow \mathrm{LUR}
$$

(ver [7]) y el autor en [6] probó que

$$
k \geq 1 \text {, Propiedad }(\mathrm{K}-\mathrm{M})+\mathrm{R} \Longleftrightarrow \mathrm{LKR} .
$$

El autor logró nuevamente generalizar la propiedad anterior e introdujo en [8], la Propiedad $(\omega \mathrm{M})$.

Definición 2.3 Se dice que el espacio de Banach E, satisface la Propiedad ( $\omega M$ ) si para todo $x \in S_{E} y\left(x_{n}\right) \subset B_{E}$ tales que,

$$
\lim _{n_{1} \ldots n_{k} \rightarrow \infty}\left\|x+\sum_{i=1}^{k} x_{n_{i}}\right\|=k+1 \quad \forall k \in \mathbb{N}
$$

entonces $\left(x_{n}\right)$ es compacto en $B_{E}$.

El autor en [14] probó que

$$
\text { Propiedad }(\omega \mathrm{M})+\mathrm{R} \Longleftrightarrow \mathrm{L} \omega \mathrm{R}
$$

y

$$
\operatorname{Prop}(1-\mathrm{M}) \Rightarrow_{\ldots} \Rightarrow \operatorname{Prop}(\mathrm{K}-\mathrm{M}) \Rightarrow \operatorname{Prop}((\mathrm{K}+1) \mathrm{M} \Rightarrow \operatorname{Prop}(\omega \mathrm{M})
$$

Ejemplo 1 Consideremos el ejemplo dado por M. A. Smith y desarrollado por el autor en [14].

En efecto, $x=\left(x^{1}, x^{2}, \ldots\right) \in\left(l_{2},\|\cdot\|_{2}\right)$ se define una norma $\|\cdot\|$ por

$$
\|x\|=\left|x^{1}\right|+\|\tilde{x}\|_{2}
$$

donde $\tilde{x}=\left(0, x^{2}, x^{3}, \ldots\right)$, que satisface, $\|x\|_{2} \leq\|x\| \leq 2\|x\|_{2}$ y por lo tanto, $\|\cdot\|$ es una norma equivalente a $\|\cdot\|_{2}$ en $l_{2}$. 
Sea $\left(\alpha_{n}\right)$ una sucesión de números reales positivos decrecientes a cero y $T: l_{2} \rightarrow l_{2}$ una aplicación lineal continua dada por

$$
T(x)=\left(\alpha_{2} x^{2}, \alpha_{3} x^{3}, \ldots\right) .
$$

Ahora, para cada $x \in l_{2}$ definimos una norma en $l_{2}$ por

$$
\left\||| x|\||=\left(\|x\|^{2}+\|T x\|_{2}^{2}\right)^{1 / 2}\right.
$$

que es equivalente a $\|\cdot\|_{2}$.

Denotemos por $E=\left(l_{2},|\|\cdot|\||)\right.$. Smith demostró que $E$ es un espacio (R), (ver [14])

T. Polak y B. Sims, (ver [14]) tomarón $\alpha_{n}=\frac{1}{n}$ y demostrarón que el espacio $E$ es $2 R$, pero no es LUR. Por tanto, tenemos que $E$ es un espacio que no satisface la Propiedad $(M)$, pués en caso contrario, como $E$ es $(R)$ entonces $E$ sería $L U R$, lo cual es falso.

Por otra parte, como $E$ es $2 R$, entonces $E$ es L2R y en consecuencia, E es LKR, para $k \geq 2$, y por lo tanto, $E$ es $L \omega R$.

De lo anterior, se obtiene que $E$ posee la Propiedad (K-M) para $k \geq 2$, y por ende, $E$ también satisface la propiedad $(\omega)$.

En conclusión, existe un espacio de Banach $(E,|| \cdot|\||)$ que posee la propiedad $(K-M)$, la propiedad ( $\omega M)$ pero no cumple la propiedad $(M)$.

En [14] el autor desarrolla un ejemplo donde se ve que, para $k \geq 1$

$$
\text { Propiedad }(\mathrm{KM}) \nRightarrow \text { Propiedad }((\mathrm{K}-1) \mathrm{M})
$$

y por tanto,

$$
\text { Propiedad }(\omega \mathrm{M}) \nRightarrow \text { Propiedad }(\mathrm{K}-\mathrm{M})
$$

Ahora, pasamos a dar nuestro principal resultado.

Teorema 2.1 Sea $(E,\|\cdot\|)$ un espacio de Banach y $F \subseteq E$ un subespacio de Chebyshev. Si $E$ posee la propiedad $\omega M$ entonces la proyección métrica $P_{F}$ es una aplicación continua.

\section{Prueba:}

Sean $x \in E$ y $\left(x_{n}\right) \subset E$ tales que $x_{n} \rightarrow x$. Veamos que $P_{F}\left(x_{n}\right) \rightarrow P_{F}(x)$.

\section{Caso 1:}

Si $x \in F$, entonces $P_{F}(x)=x$ y por tanto se cumple que,

$$
\begin{gathered}
\left\|P_{F}(x)-P_{F}\left(x_{n}\right)\right\|=\left\|x-P_{F}\left(x_{n}\right)\right\| \leq\left\|x-x_{n}\right\|+\left\|P_{F}\left(x_{n}\right)-x_{n}\right\| \\
\leq\left\|x-x_{n}\right\|+d\left(x_{n}, F\right) \leq 2\left\|x-x_{n}\right\| \rightarrow 0, n \rightarrow \infty,
\end{gathered}
$$

así, $P_{F}\left(x_{n}\right) \rightarrow P_{F}(x)$, lo cual nos muestra que $P_{F}$ es una aplicación continua.

\section{Caso 2:}

Si $x \notin F$, entonces $d(x, F)>0$. Sean

$$
\widetilde{x}_{n}=\frac{x_{n}-P_{F}(x)}{\left\|x_{n}-P_{F}(x)\right\|} ; \quad \widetilde{x}_{n}=\frac{x-P_{F}(x)}{\left\|x-P_{F}(x)\right\|}
$$


entonces, $\widetilde{x}_{n} \rightarrow \widetilde{x}$, y además se cumple que,

$$
P_{F}\left(x_{n}\right) \rightarrow P_{F}(x) \Longleftrightarrow P_{F}\left(\widetilde{x}_{n}\right) \rightarrow P_{F}(\widetilde{x})=0 .
$$

Podemos asumir que $x \in S_{E}$ y $P_{F}(x)=0$. Veamos que $P_{F}\left(x_{n}\right) \rightarrow 0$. De nuevo, dos opciones se nos presentan; primero: $P_{F}\left(x_{n}\right) \rightarrow m$. Entonces

$$
\begin{gathered}
\|x\|=\left\|x-P_{F}(x)\right\| \leq\|x-m\| \leq \lim _{n \rightarrow \infty}\left\|x_{n}-P_{F}\left(x_{n}\right)\right\| \\
\leq \lim _{n \rightarrow \infty}\left\|x_{n}\right\|=\|x\|,
\end{gathered}
$$

así, $\|x\|=\|x-m\|$ y por la unicidad del punto cercano, $m=0$.

Segundo: $P_{F}\left(x_{n}\right) \nrightarrow \rightarrow 0$. Entonces existe una subsucesión $\left\{P_{F}\left(x_{n_{i}}\right)\right\}$ de $\left\{P_{F}\left(x_{n}\right)\right\}$, la cual, sin pérdida de generalidad, la podemos tomar como $\left\{P_{F}\left(x_{n}\right)\right\}$, tal que para algún $\varepsilon_{o}>0$ y todo $m, n$ se tiene

$$
\left\|P_{F}\left(x_{n}\right)-P_{F}\left(x_{m}\right)\right\|>\varepsilon_{o} .
$$

Como,

$$
\begin{gathered}
\|x\| \leq\left\|x-P_{F}\left(x_{n}\right)\right\| \leq\left\|x_{n}-P_{F}\left(x_{n}\right)\right\|+\left\|x-x_{n}\right\| \leq\left\|x_{n}\right\|+\left\|x_{n}-x\right\|, \\
\lim _{n \rightarrow \infty}\left\|x_{n}-x\right\|=0 \quad \text { y } \lim _{n \rightarrow \infty}\left\|x_{n}\right\|=\|x\|
\end{gathered}
$$

entonces se concluye que,

$$
\lim _{n \rightarrow \infty}\left\|x-P_{F}\left(x_{n}\right)\right\|=\|x\|=1 .
$$

De esta última afirmación y teniendo presente que

$$
\|x\| \leq\left\|x-P_{F}\left(x_{n}\right)\right\|
$$

se sigue

$$
\begin{aligned}
k+1=(k+1)\|x\| & =\|(k+1) x\|=\|x+k x\| \\
& \leq\left\|x+k x-\sum_{i=1}^{k} P_{F} x_{n_{i}}\right\| \\
& =\| x+\sum_{i=1}^{k}\left(x-P_{F}\left(x_{n_{i}}\right) \|\right. \\
& \leq\|x\|+\sum_{i=1}^{k}\left\|x-P_{F}\left(x_{n_{i}}\right)\right\|
\end{aligned}
$$

y haciendo, $n_{1}, \ldots, n_{k} \rightarrow \infty$ obtenemos

$$
k+1 \leq \lim _{n_{1} \ldots n_{k} \rightarrow \infty}\left\|x+\sum_{i=1}^{k}\left(x-P_{F} x_{n_{i}}\right)\right\| \leq k+1,
$$


y por lo tanto

$$
\lim _{n_{1} \ldots n_{k} \rightarrow \infty}\left\|x+\sum_{i=1}^{k}\left(x-P_{F} x_{n_{i}}\right)\right\|=k+1, \quad \forall k \in \mathbb{N}
$$

Ya que $E$ satisface la propiedad $\omega M$, entonces $\left\{x-P_{F}\left(x_{n}\right)\right\}$ es compacto en $B_{E}, \mathrm{y}$ por tanto posee una subsucesión convergente, lo cual contradice a $(*)$. Así tenemos que $P_{F}\left(x_{n}\right) \rightarrow 0$, y esto nos muestra que $P_{F}$ es una aplicación continua.

El siguiente resultado nos muestra la generalización anteriormente anunciada.

Corolario 2.1 Sean $(E,\|\cdot\|)$ un espacio de Banach y $F \subset E$ un subespacio de Chebyshev. Entonces, $P_{F}$ es una aplicación continua en cualquiera de los siguientes casos:

1. E es un espacio $L N U C$

2. E es un espacio $L \omega U C$

3. E es un espacio $L \omega R$.

\section{Prueba:}

En [5] se muestra que $L \omega U R \Longrightarrow L N U C$ y en [6] mostramos que

$$
L N U C \Longrightarrow \text { Propiedad }(\omega M) \text {. }
$$

Además, en [7] mostramos que $L-k R \Longrightarrow \operatorname{Propiedad}(k-M)$, pero esta afirmación es cierta para todo $k \in \mathbb{N}$ por tanto se cumple que $L \omega R \Longrightarrow \operatorname{Propiedad}(\omega M)$.

Así, tenemos que en cualquiera de los tres casos se cumple que la proyección métrica $P_{F}$ es una aplicación continua.

\section{Agradecimiento}

Doy mis más expresivas gracias al Mathematical Institute, Slovak Academy of Sciences, Bratislava - Slovakia por su colaboración prestada durante mi estadía en el Instituto y en especial al Profesor I. Dobrakov por sus atenciones para con el autor.

Gracias al Grupo de Análisis Funcional por la ayuda financiera para reescribir este trabajo.

Muy agradecido a los evaluadores por sus observaciones para mejorar el presente trabajo. 


\section{Referencias}

[1] Brown, A.L. (1974) "A Rotund reflexive space having a subspace of Codimension two with a discontinous metric projection", Mich. Math. J. 21: 145-151.

[2] Fitzpatrick, S. (1980) "Metric projections and the diffentiability of distance functions", Bull. Austral. Math. Soc. 22: 291-312.

[3] Giles, J. R. (1982) Convex analysis with application in differentiation of Convex functions. Pitman Research Notes in Mathematics $\mathbf{5 8 .}$

[4] Holmes, R. A Course on optimization and Best approximation. Lecture Notes in Mathematics, 257, Springer-Verlag.

[5] Bor-Luh Lin; Zhang, W. (1991) Some Geometric Properties Related to Uniform Convexity of Banach Spaces. Functions Spaces. Lecture Notes in Pure and Appl. Math., Marcel Decker, 136: 281-294.

[6] Morales, J. R. (1992) "La Propiedad $(k-M)$ en espacios de Banach", Notas de Matemáticas ULA, 118.

[7] Morales, J. R. (1992) "Sobre los espacios k-M", Revista Colombiana de Matemáticas 26: $115-120$.

[8] Morales, J. R. "Una nota sobre los espacios $L \omega R$ ". Por aparecer.

[9] Narang, T.D. (1977) "Convexity of Chebyshev Sets", Nic. Arc. V. W. 3, XXV: 377402.

[10] Sullivan, F. (1979) "A generalization of uniformly rotund Banach spaces", Can. J. Math. 31: 628-636.

[11] Yu Xintai (1985) "On LkUR spaces", Chin. Ann of Math. 6B(4): 465-469.

[12] Holmes, R.; Kripke, B. (1968) "Smoothness of approximation", Michigan Math. J. 15: $225-248$.

[13] Nan-Chao; Wang-Jian Hua (1988) "On the LK-UR y L-KR spaces", Math. Proc. Camb. Phili. Suc. 104: 521-526.

[14] Morales, J.R. "Los espacios L $\omega R$ ". Por aparecer. 